\title{
MODELING OF LAMINAR FLOW TUBULAR REACTOR USING VELOCITY PROFILE
}

\author{
Vinod Kallur ${ }^{1}$, M A Lourdu Antony Raj ${ }^{2}$ \\ ${ }^{I}$ Associate Professor, Chemical Engineering Department, $R$ V College of Engineering, Bangalore, India, \\ ${ }^{2}$ Professor, Chemical Engineering Department, $R$ V College of Engineering, Bangalore, India
}

\begin{abstract}
Design of reactor using traditional performance equations is inadequate as it does not take into account non-ideality in the reactors. Quantification of non-ideality is done using Residence Time Distribution studies (RTD). But RTD studies can be carried out on already existing reactor. Therefore this technique will not help in incorporating non-ideality in the design stage. Another method is to use Computational Fluid Dynamics (CFD) packages and predict RTD. The methods used to predict reactor performance using RTD involves modeling, fitting the RTD data to the model to determine the model parameters such as number of tanks in tanks in series model or dispersion number in axial dispersion model. These models are inadequate as the RTD may not be a proper fit. After the model fit, using the model parameter to determine the performance of the reactor is cumbersome and in some cases it is not possible. There are several models that predict reactor performance which use Convection - DiffusionReaction (CDR) equations and these methods are computationally intensive. Simpler models that are less computationally intensive but adequate enough to predict reactor performance are to be developed. In the present paper a model is developed which makes use of only velocity profile to predict homogeneous reactor performance. Algorithm of the model is then applied to Laminar Flow Tubular Reactor (LFTR). Testing of the model is done by comparing the results of the model with those predicted by well established analytical methods for LFTR. The results of the model and those of LFTR agree well.
\end{abstract}

Keywords: Laminar Flow, velocity profile, reactor, conversion, grid cell, CSTR ****

\section{INTRODUCTION}

Reactor modeling that incorporates non-ideality at the design stage requires better understanding of mixing that occurs in the reactor. In the classical reactor design, only the mass conservation principle is used without taking into account how exactly reaction takes place in the reactor. Mass conservation principle along with certain assumptions is used to develop design procedures. Such reactors are called ideal reactors. Design equations developed are called performance equations. Though mixing contributes to the reaction, it does not play any role in the performance equations. The ideality and its validity play an important role in the accuracy of performance prediction of the reactors using these equations. The simplest way of quantifying mixing adequate enough to incorporate kinetics in the evaluation of reactor is conventionally carried by using Residence Time Distribution (RTD) studies [1].

Unfortunately, RTD is not available at the design stage. RTD study is conducted on the existing reactors. There is no way RTD can be used at the design stage except the cases where RTD can be simulated using appropriate model. However there are no generic RTD models applicable to all reactors. Low dimensional mixing models such as axial dispersion recycle and tanks in series models are simple to use. These models are single parameter models [2] and cannot be fitted to all reactors. Whenever single parameter models are inadequate, multi-parameter models may be used [1]. Determination of model parameters can pose difficulties. Another approach is to use of Computational Fluid Dynamics to analyze mixing effect on reactor performance by numerically solving the ConvectionDiffusion-Reaction (CDR) equations. This is computationally intensive. For this reason the low dimensional models are very popular and are used extensively despite their inherent fallibility [3].

Hydraulics and RTD can be affected by many factors such as impeller geometry and shape, impeller speed, baffle dimensions. Inappropriate RTD sampling and numerical diffusions can lead to significant deviations in the RTD predictions using CFD [4]. Advancements in CFD and precision in measurements have significantly improved the knowledge of the flow fields in the reactors. Velocity fields can easily be obtained from 3D CFD simulations. However, an effective method for quantifying spatial non-uniformity of mixing using such velocity fields is still missing. Measuring or computing flow pattern is helpful for some understanding of the non-uniform mixing but no quantitative mixing measures can be obtained directly from velocity fields. RTD theory also cannot provide a definitive answer on the size, locations, and intensity of the non-uniformity [5].

Mixing in reacting flows forms a technologically important class of operations in all process industries. Laminar flow regime is predominant in polymer and food processing, as often it involves viscous liquids. Modeling of the flow in the presence of chemical reaction presents a greater challenge and has been studied extensively in the last 50 years [6]. The RTD of a system represented by a rigid pipe and laminar flow is a specific case typical for the food industry, for flow in micro-channels, in meso- reactors and micro-reactors [7]. 
Modeling of mixing processes in deterministic laminar flows has not received much attention, and detailed description of such flows is still lacking, though utility of these systems is significant [8]. The models that demand moderate computational time and still accurate enough to be practically useful are to be developed [9].

Though the laminar flow is deterministic, not much work is available in the literature exploiting its utility. In cases where diffusion is not the dominating factor contributing to reactor performance, mathematically simpler models are to be formulated and tested. The present work deals with formulation and testing of a model for Laminar Flow Tubular Reactors.

\section{THE FORMULATION OF THE MODEL}

Laminar flow is mathematically deterministic. Velocity profile in the tubular reactor is well known. Further flow of the reacting mixture occurs in a sequential manner. This is used to develop the model to predict the reactor performance.

\subsection{Back Ground of the Model}

The model in the present work uses predetermined velocity profile. The reactor is assumed to be made up of several 3-D cells and each cell acts as an ideal Continuous Stirred Tank Reactor (CSTR). Since the velocity vectors around this cell are known, it is possible to determine the volumetric flow rate into the cell. Knowing the volume of the cell, the spacetime for CSTR can be determined. Applying performance equation of CSTR (it is an algebraic equation and therefore simple) and knowing feed information, the composition at the exit can be determined. This is the principle used in the present model. Since there is no parameter involved, the model may be referred as zero-parameter model.

As a proof of principle, laminar flow tubular reactor (LFTR) is used. The velocity profile for LFTR is mathematically known. Further, there are well established models to determine the conversion in these reactors though applicable to selected kinetics. These models determining conversion in LFTRs make use of segregated flow model [1]. For some kinetics analytical solutions are available and others require numerical solutions. All these ideas are used to test the principle that 3-D grid cells in the reactor can be assumed to be ideal CSTRs and using this, the performance of the reactor can be determined.

The LFTR in cylindrical coordinates is assumed to be made up of 3-D cells which are formed by slices (sections in axial direction or z-direction), sectors (sections in $\theta$ direction) and concentric circles (in radial locations) as shown in Fig-1.
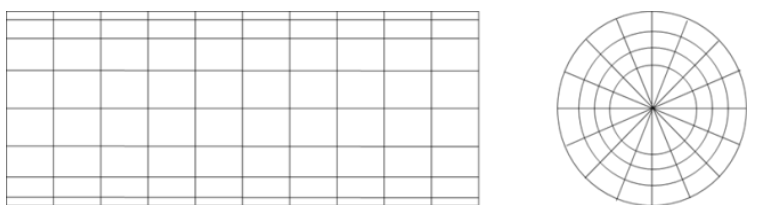

Fig- 1: Schematic of 3-D cells
Each 3-D cell is assumed to be an ideal CSTR. This model is different from tanks in series model. Tanks in series model make use of equal volume slices which are treated as CSTRs connected in series. However model proposed in the present work treats LFTR as series of CSTRs necessarily not of equal volume connected in series. Further, in contrast to tanks in series model, the proposed model will have several CSTRs in radial direction as well. There is no interaction between cells (CSTRs) in radial direction as there is no flow in that direction in LFTR. Mathematically CSTR requires only solution of algebraic equations and reduces computational time. The solution is obtained in a sequential manner starting from the cell into which the reactants are entering and ending at the cells from where the product stream comes out.

\subsection{Assumptions}

The flow in the reactor is laminar.

1. The velocity profile is parabolic throughout the reactor. The end effects are neglected.

2. The diffusion effects are negligible.

3. The physical properties of reaction mixture are constant i.e., do not vary with composition.

4. The reaction is of constant volume.

5. The condition within the reactor is isothermal.

\subsection{Algorithm of the Model}

1. Input of values: Inlet concentration of A and B in individual streams, rate constant, diameter and length of the reactor, volumetric flow rate of streams $\mathrm{A}$ and $\mathrm{B}$, number of slices, concentric circles and sectors.

2. Calculations will start from the face at the entrance which is outer-most in the radial direction as shown shaded in the Fig-2.

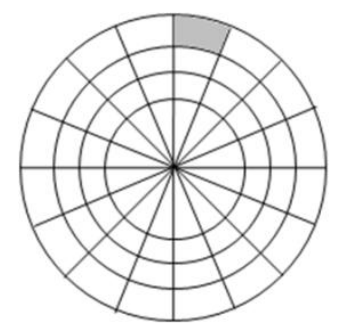

Fig- 2: Face of an outermost elemental CSTR

3. Geometric Centre of each elemental flow area subtending an of angle $2 \pi / r$ at the center is located using

$$
r_{i}=R-(2 i-1) \Delta r
$$

where $\Delta r=\frac{R}{2 q}, i=1,2,3 \ldots, q$

$\mathrm{q}$ is number of concentric circles, $i$ refers to a grid cell and $R$ is the radius of the tubular reactor 
The measurement of $r_{i}$ and $\Delta r$ are shown in Fig-3.

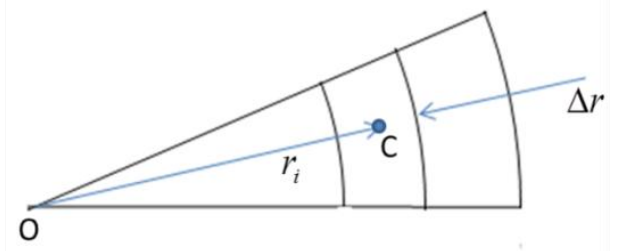

Fig- 3: Location of geometric centre

4. Flow area on its surface is given by

$A_{i}=\frac{4 \pi}{r} r_{i} \Delta r$

where $r$ is the number of sectors.

5. Volume of the cell Vi is given by

$$
V_{i}=A_{i} L / p
$$

where $p$ is the number of slices.

6. Local velocity at the center of the cell [6] is given by

$$
v_{z i}=2 \bar{v}_{z}\left[1-\left(\frac{r_{i}}{R}\right)^{2}\right]
$$

where $v_{z i}$ is the velocity at the center of $i^{t h}$ cell and $\bar{v}_{z}$ is the average velocity of the reacting liquid in the tubular reactor.

7. Volumetric flow rate through this cell is determined as product of local velocity at the center and flow area determined in step 4 and is given by

$$
Q_{i}=v_{z i} A_{i}
$$

8. Space-time for this grid cell is determined as

$$
\tau_{i}=\frac{V_{i}}{q_{i}}
$$

9. Conversion in the grid cell assuming it to be an ideal CSTR [1] is given by

$$
X_{A i}=\frac{\tau_{i}\left(-r_{A i}\right)}{C_{A 0 i}}
$$

The value of $\left(-r_{A i}\right)$ is to be evaluated at the composition prevailing at the exit of the grid cell. Composition at the exit will be the initial values for the next grid cell in the direction of flow.
10. Calculations are repeated until the exit grid cell is reached.

11. Procedure is repeated for the next inner radial position starting from step 3 .

12. For the innermost gird cell as per this algorithm, $r_{i}-\Delta r$ in the equation of step 4 will become zero. The shape of this grid cell will be cylindrical.

13. These calculations are done only for one sector. Due to symmetry, the composition at a given radial position and at a given cross section is constant.

14. Average concentration at the exit of the reactor [7] is determined as

$$
\bar{C}_{A}=\frac{\sum_{\text {one sector }} C_{A i} Q_{i}}{\frac{\pi}{r} R^{2} \cdot \bar{v}_{z}}
$$

Since it is computationally intensive, a computer program was written and tested. After extensive testing of the program, the model testing was carried out. The model testing required several modifications to the program in a progressive manner. The final program is generic which uses analytical methods whenever applicable and uses numerical methods otherwise.

The proposed model is tested using other well established methods [1]. The testing methods used are computationally intensive. However the proposed model uses simple CSTR equation which is algebraic. Only for higher order equations, the model uses numerical methods.

\section{MANUAL TESTING OF THE PROGRAM}

Taking less number of cells (slices 2, circles 2 and sectors 4 making total 16 cells), manual calculations were done using the values listed in Table-1. Fig-4 gives cross section of the reactor with the cells. Table- 2 gives the results of manual calculations.

Table-1: Values used for algorithm testing

\begin{tabular}{|l|l|l|}
\hline No & Input variable & Value \\
\hline 1. & $\mathrm{D}$ & $1 \mathrm{~m}$ \\
\hline 2. & $\mathrm{~L}$ & $5 \mathrm{~m}$ \\
\hline 3. & $\mathrm{p}$ & 2 \\
\hline 4. & $\mathrm{q}$ & 2 \\
\hline 5. & $\mathrm{r}$ & 4 \\
\hline 6. & $v_{A}$ & $0.0008 \mathrm{~m}^{3} / \mathrm{s}$ \\
\hline 7. & $v_{B}$ & $0.0008 \mathrm{~m}^{3} / \mathrm{s}$ \\
\hline 8. & $C_{A 0}^{\prime}$ & $70 \mathrm{~mol} / \mathrm{m}^{3}$ \\
\hline 9. & $C_{B 0}^{\prime}$ & $70 \mathrm{~mol} / \mathrm{m}^{3}$ \\
\hline 10. & $\mathrm{k}$ & $\left.0.0002 \mathrm{~mol}^{2} / \mathrm{m}^{3} . \mathrm{s}\right)$ \\
\hline
\end{tabular}




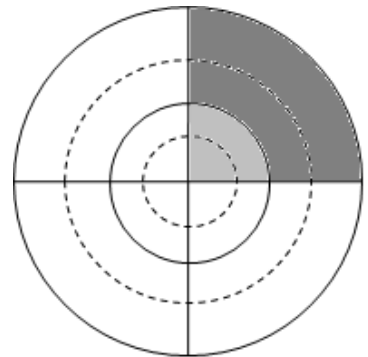

Fig-4: Cross sectional view with $p=2, q=2$ and $r=4$

The results obtained in manual calculations were compared with the intermediate corresponding values while program was being run. There was excellent agreement between the two.

The model is found to be adequate when the number of grid cell is very small (16 in total). This kind of testing for large number of cells is very time consuming, as it involves large numerical calculations. For higher number of cells, a spreadsheet program was used and agreeing results were obtained.

Table-2: Values obtained in manual calculations as per the algorithm

\begin{tabular}{|c|c|c|c|c|}
\hline Slice No. & Slice 1 & Slice 2 & Slice 1 & Slice 2 \\
\hline Variable & \multicolumn{2}{|c|}{$\begin{array}{l}\text { Outer Annular } \\
\text { Ring in one } \\
\text { sector }\end{array}$} & \multicolumn{2}{|c|}{$\begin{array}{l}\text { Inner Circle in } \\
\text { one sector }\end{array}$} \\
\hline$r_{i}$ & \multicolumn{2}{|l|}{0.375} & \multicolumn{2}{|l|}{0.125} \\
\hline$A_{i}$ & \multicolumn{2}{|l|}{0.1472} & \multicolumn{2}{|l|}{0.04908} \\
\hline$v_{z}$ & \multicolumn{2}{|c|}{$1.782 \times 10^{-3}$} & \multicolumn{2}{|c|}{$3.819 \times 10^{-3}$} \\
\hline$V_{i}$ & \multicolumn{2}{|l|}{0.368} & \multicolumn{2}{|l|}{0.1227} \\
\hline$q_{z}$ & \multicolumn{2}{|c|}{$2.62 \times 10^{-4}$} & \multicolumn{2}{|c|}{$38.19 \times 10^{-4}$} \\
\hline$\tau_{i}$ & \multicolumn{2}{|l|}{1402.5} & \multicolumn{2}{|l|}{654.59} \\
\hline$X_{A i}$ & 0.7277 & 0.5474 & 0.6293 & 0.4725 \\
\hline$C_{A i}$ & 9.53 & 4.313 & 12.971 & 6.8418 \\
\hline$\sum C_{A i} Q_{i}$ & \multicolumn{2}{|c|}{$1.1318 \times 10^{-3}$} & \multicolumn{2}{|c|}{$1.2821 \times 10^{-3}$} \\
\hline$\sum Q_{i}$ & \multicolumn{4}{|c|}{$0.4498 \times 10^{-3}$} \\
\hline$\overline{C_{A}}$ & \multicolumn{4}{|l|}{5.3655} \\
\hline$X_{A}$ & \multicolumn{4}{|l|}{0.84669} \\
\hline$X_{A I}$ & \multicolumn{4}{|l|}{0.945} \\
\hline
\end{tabular}

\section{OPTIMIZATION OF NUMBER OF GRID}

\section{CELLS}

Optimum number of slices, sectors and concentric circles that give converged conversion was determined by increasing the number of grid cells systematically. The conversion is expected to get more and more accurate as the number of grid cells increase or the size of the grid cells is smaller. However with increased number of grid cells it takes more processing time. There is a need to determine an optimum number of grid cells without compromising on the accuracy.

For optimizing the number grid cells number of slices, sectors and circles was kept at 5 each and gradually increased. Values of input variables used for optimization of number of grid cells are given in Table-3. Model conversion as function of number of sectors, circles and sectors are depicted in Chart -1, Chart-2 and Chart-3.

It is found that the conversion is least sensitive to number of sectors and most sensitive to number of slices. It is also evident that the conversion converges from higher value in case of increasing number of circles whereas it converges from lower value in case of increasing number of slices.

Table 3 Parameter values for optimizing grid cells

\begin{tabular}{|c|c|c|}
\hline No & Parameter & Value \\
\hline 1 & $\mathrm{~L}$ & 5 \\
\hline 2 & $\mathrm{D}$ & 1 \\
\hline 3 & $\mathrm{v}_{\mathrm{A}}$ & 0.0008 \\
\hline 4 & $\mathrm{v}_{\mathrm{B}}$ & 0.0008 \\
\hline 5 & $\mathrm{k}$ & 0.001 \\
\hline 6 & $\mathrm{C}_{\mathrm{A} 0}$ & 10 \\
\hline 7 & $\bar{C}_{\mathrm{B} 0}$ & 10 \\
\hline
\end{tabular}

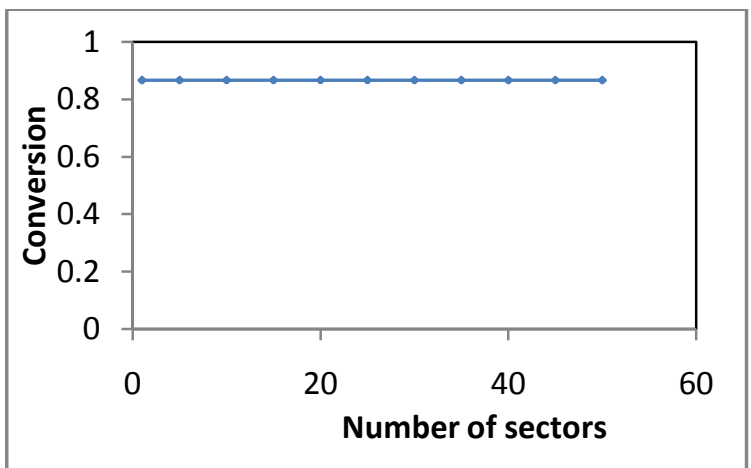

Chart -1: Conversion vs. number of sectors

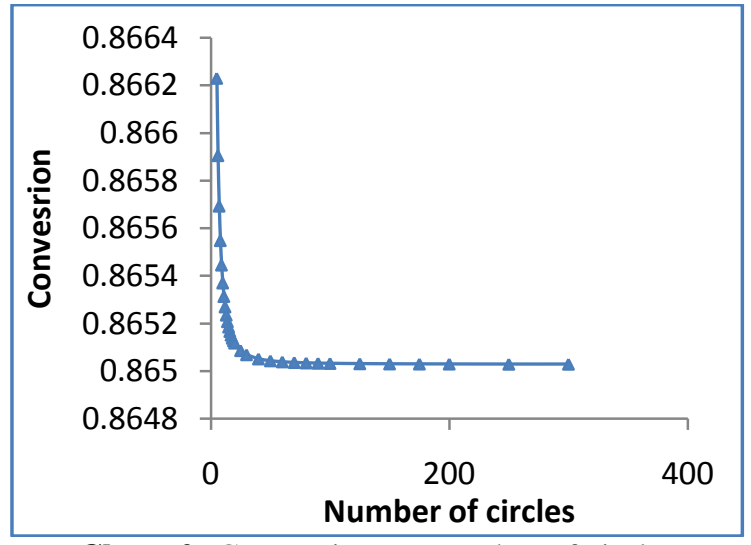

Chart-2: Conversion vs. number of circles 


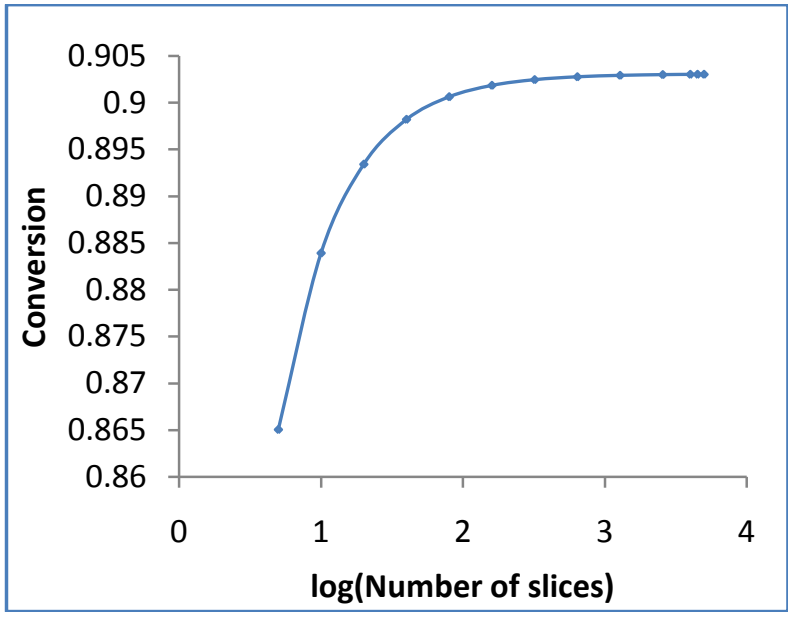

Chart-3: Conversion vs. log (number of slices).

Though the conversion converges to a single value for lesser number of grid cells, the optimum number of grid cells was taken corresponding to be $p=5000, q=200$ and $r=50$.

\section{ANALYTICAL TESTING OF THE MODEL}

The model was tested using independent analytical method that is used to determine conversion in LFTR. The conversion in LFTR [1] for second order reactions is given by

$\frac{\bar{C}_{A}}{C_{A 0}}=1-k C_{A 0} \bar{t}\left[1-\frac{k C_{A 0} \bar{t}}{2} \ln \left(1+\frac{2}{k C_{A 0} \bar{t}}\right)\right]$

where $\bar{t}$ is mean residence time, $\bar{C}_{A}$ is average concentration of reactant $\mathrm{A}$ at the exit.

The model conversion and conversion determined using Equation 9 (Laminar Conversion) for $C_{A 0}=C_{B 0}=75$ is shown in Chart-4.

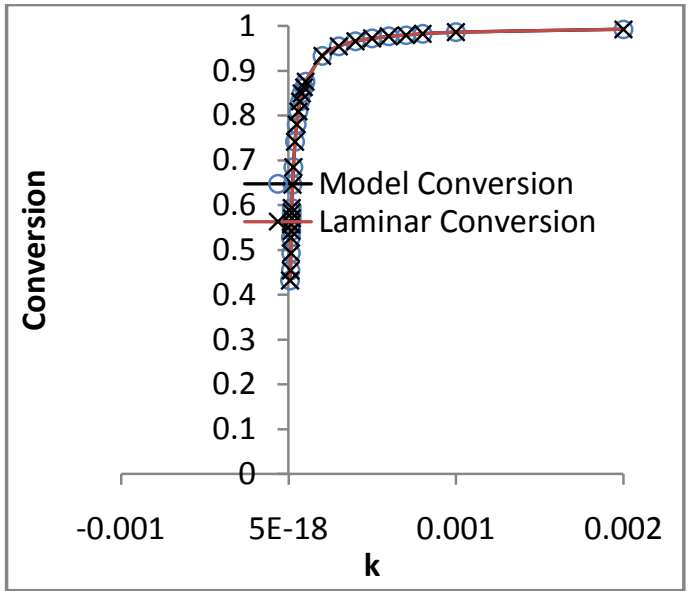

Chart- 4: Comparison of model and laminar conversion

From Chart-4, it is clear that model conversion completely agree with laminar flow conversion. This validates the model for bimolecular reaction with equimolar feed. The model and laminar conversions were determined for various values of initial concentrations and in all the cases the results validated the model. For different values of initial concentrations the difference between model conversion and ideal conversion vs. $k$ is depicted in Chart-5.

It is observed that there is single maximum difference between 0.05 and 0.055 in conversions for all values of $k$ and all values of initial concentrations. With increasing initial concentration, the value of $k$ where maximum occur decreases. This was further analyzed in terms of Damkohler number, $N_{D a}$.

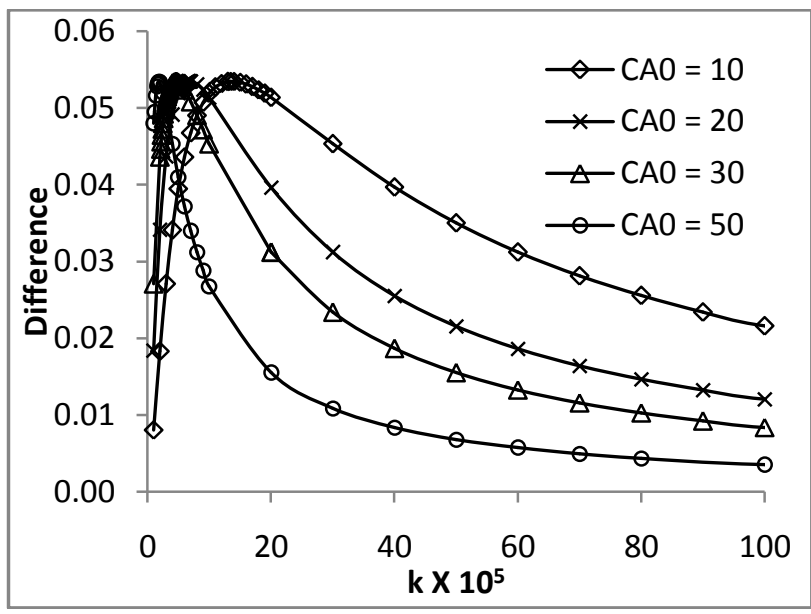

Chart-5: Variation of difference in conversion with $k$

A peculiar trend was observed when the difference between and ideal and model conversions were plotted against Damkohler number and the plot is given in Chart-6. The maximum difference occurred for all concentrations at one single value of Damkohler number less than 2. Theoretical basis for difference between model conversion and ideal conversion $(\Delta \mathrm{X})$ occurring at a single $N_{D a}$ has also been investigated. It was found that theoretical basis also gives maximum at a single value of $N_{D a}$.

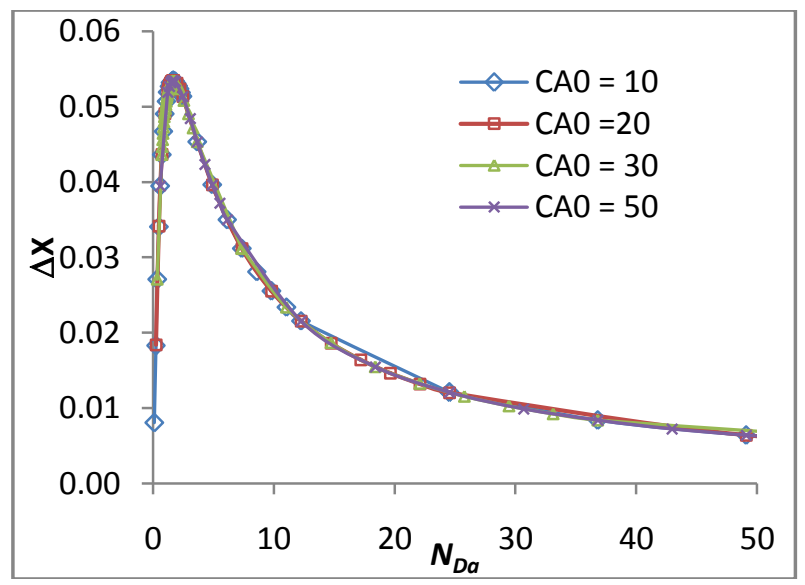

Chart-6: Difference between model and ideal conversion vs. $N_{D a}$. 


\subsection{Theoretical Basis for maximum $\Delta X$ vs. NDa}

The difference between model and ideal conversion can be written as

$$
\Delta X=X_{\text {A.Ideal }}-X_{\text {A.Model }}
$$

Since the model and laminar conversions are same as shown in Chart-4 are same, Equation 10 can be written as

$$
\Delta X=X_{\text {A.Ideal }}-X_{\text {A.Laminar }}
$$

For bimolecular reaction with equimolar feed, Equation 11 becomes

$$
\Delta X=\frac{N_{D a}}{1+N_{D a}}-N_{D a}\left[1-\frac{N_{D a}}{2} \ln \left(1+\frac{2}{N_{D a}}\right)\right]
$$

Maximum difference in $\Delta X$ is determined by setting

$$
\begin{aligned}
& \frac{d(\Delta X)}{d N_{D a}} \text { to zero to get } \\
& \frac{1}{1+N_{D a}}-1-\frac{N_{D a}}{2+N_{D a}}+N_{D a} \ln \left(1+\frac{2}{N_{D a}}\right)=0
\end{aligned}
$$

From this we get $N_{D a}=1.6825$. Substituting this value in Equation 12, the maximum $\Delta X$ is found to be 0.0534 . This is the value of $N_{D a}$ where all maxima coincide as shown in Chart-6.

\section{CONCLUSIONS}

A model was developed to determine the performance of Laminar Flow Tubular Reactor and computer program was written and tested. The results of the model were compared to those obtained by various well established analytical methods. In all the methods of testing and validation used, the results agree well.

This establishes that the velocity profile can be successfully used to predict the performance of LFTR with excellent accuracy. Whenever the reaction is such that the diffusion effects are negligible, this model may be used. Testing in this paper is carried out for simple kinetics (bimolecular reaction with equimolar feed) for which analytical solution for testing is available in the literature. Analytical testing of the model cannot be done for bimolecular reaction with nonequimolar feed. The model is to be further tested and validated for general kinetics.

\section{REFERENCES}

[1] Octave Levenspiel, Chemical Reaction Engineering, John Wiley \& Sons, $3^{\text {rd }}$ Edition, 1999.

[2] Saikat Chakraborty, Vemuri Balakotaiah, A novel approach for describing mixing effects in homogeneous reactors, Chemical Engineering Science, 58 (2003) pp, 1053-1061.

[3] Saikat Chakraborty, Vemuri Balakotaiah, Madhuchhanda Bhattacharya, Michael P. Harold, Vemuri Balakotaiah, Low dimensional models for homogeneous stirred tank reactors, Chemical Engineering Science, 59, 2004, pp 5587-5596.

[4] Hua Bai, Amber Stephenson, Jorge Jimenez, Dennis Jewell, Paul Gillis, Modeling flow and residence time distribution in an industrial-scale reactor with plunging jet inlet and optional agitation, Chemical Engineering research and Design, 86, 2008, pp 1462-1476.

[5] Minye Liu, Age distribution and the degree of mixing in continuous flow stirred tank reactors Home literature, Chemical Engineering Science, 69, 2012, pp 382-393.

[6] Saikat Chakraborty, Vemuri Balakotaiah, Low dimensional models describing mixing effects in laminar flow tubular reactors, Chemical Engineering Science, 57, 2002, pp 2545-2564.

[7] H. Chlup, P. Novotny, R. Zitny, International Journal of Heat and Mass Transfer, 55, 2012, pp 6458-6462.

[8] P. E. Arratia, J. P. Lacombe, T. Shinbrot, F. J. Muzzio, Segregated regions in continuous laminar stirred tank reactors, Chemical Engineering Science, 59, 2004, pp 1481-1490.

[9] Lene Kristin Hjertager Osenbroch, Experimental and Computational Study of Mixing and Fast Chemical Reactions in Turbulent Liquid Flows, PhD Thesis, Aalborg University, 2004.

[10] R. Byron Bird, Warren E. Stewart, Edwin N. Lightfoot, Transport Phenomena, John Wiley \& Sons, 2002.

\section{BIOGRAPHIES}

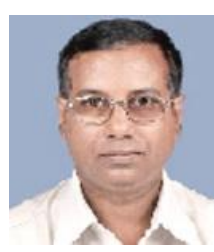

Vinod Kallur obtained his BE and M.Tech in Chemical Engineering from KREC (NITK) Surathkal, India. He has 20 years of teaching experience. His interests include reaction engineering, computational chemical engineering and modeling. Presently he is Associate Professor in Chemical Engineering Department of $\mathrm{R} \mathrm{V}$ College of Engineering, Bangalore, India.

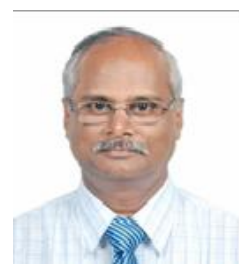

Dr M.A.Lourdu Antony Raj obtained his BE in Chemical Engineering from Annamalai University, India in 1981 and $\mathrm{PhD}$ from Bangalore University. His area of research includes reaction engineering, transfer operations and modeling. He served as Head, Chemical Engineering Department at R V College of Engineering, Bangalore, India during 2004-2010. Presently he is Dean, Student affairs. He has 31 years of teaching and 16 years of research experience. He has more than 20 national and international publications and 18 national and international conference publications. 